\title{
Cervical Spondylosis Management through the Spactacles of Homoeopathy
}

\author{
Shashi Bhushan Singh ${ }^{1}$, Ravi Prakash ${ }^{2}$ \\ ${ }^{1}$ Assistant Prof. Department of Obs. \& Gynae., R.B.T.S Govt. Homoeopathic Medical College \& Hospital, \\ Muzaffarpur, Bihar \\ ${ }^{2}$ Associate Prof. Department of Materia-Medica, R.B.T.S Govt. Homoeopathic Medical College \& Hospital, \\ Muzaffarpur, Bihar
}

Correspondence should be addressed to Dr. Ravi Prakash,drravinih@gmail.com

Publication Date: 9 September 2021

DOI: https://doi.org/10.23953/cloud.ijaayush.506

Copyright (C 2021 Dr. Shashi Bhushan Singh, Dr. Ravi Prakash. This is an open access article distributed under the Creative Commons Attribution License, which permits unrestricted use, distribution, and reproduction in any medium, provided the original work is properly cited.

Abstract Cervical spondylosis is one of the commonly seen diseases nowadays. Neck pain, which usually arises from diseases of the cervical spine \& soft tissues of the neck, is common. "SPONDYLO" is a Greek term, meaning "Vertebra" \& spondylosis generally mean changes in the vertrbral joint characterized by increasing degeneration of the intervertebral disc with subsequent changes in the bones \& soft tissue. The management of cervical spondylosis is very much effective with Homoeopathic medicine. This article stress upon the risk factor, pathophysiology, symptomatology, investigation in concise manner as well as emphasises how homoeopathy can manage in such cases by its holistic approach of treatment.

Keywords Cervical spondylosis, Case-taking, Homoeopathic medicine

\section{Introduction}

Neck pain is one of the most common problems that one encounters in day to day life. It is probably as common as common cold. Cervical spondylosis may be caused by one or more of several complaints. A very common mistake for cervical spondylosis is to perceive the cause of illness as a singular factor. Treating the patient for a single factor like a spur seen on X-ray or a slipped disc in the neck seen on a myelogram or CT.

Cervical spondylosis tends to develop after the age of 30 , and becomes more common with increasing age. The underlying cause is the age-related degeneration ('wear \& tear') of the vertebrae and discs in the neck region.

As the 'discs' degenerate, over many years they become thinner. Sometimes the vertebrae develop small, rough areas of bone on their edges. The nearby muscles, ligaments, and nerves may become irritated by these degenerative changes which can cause troublesome symptoms. The most commonly effected nerve roots are C5, C6, C7. 


\section{Risk Factor}

Cervical spondylosis is caused by chronic wear on the cervical spine.

a) Age: By the age of 50 years, it effects $50 \%$ of the population.

b) Sex: In males, the prevalence was $13 \%$ in $3^{\text {rd }}$ decade to $100 \%$ by 70 years. It occur in younger due to sudden protussion of cervical disc

c) Trauma

d) Work activity: It is more higher in office worker due to long hours looking down while working

e) Genetic

\section{Pathophysiology}

Cervical spondylosis is the degeneration of the intervertebral disc. When we get older, the disc fragment loose water content \& collapse. Intervertebral disc loose its elasticity \& compress each other.

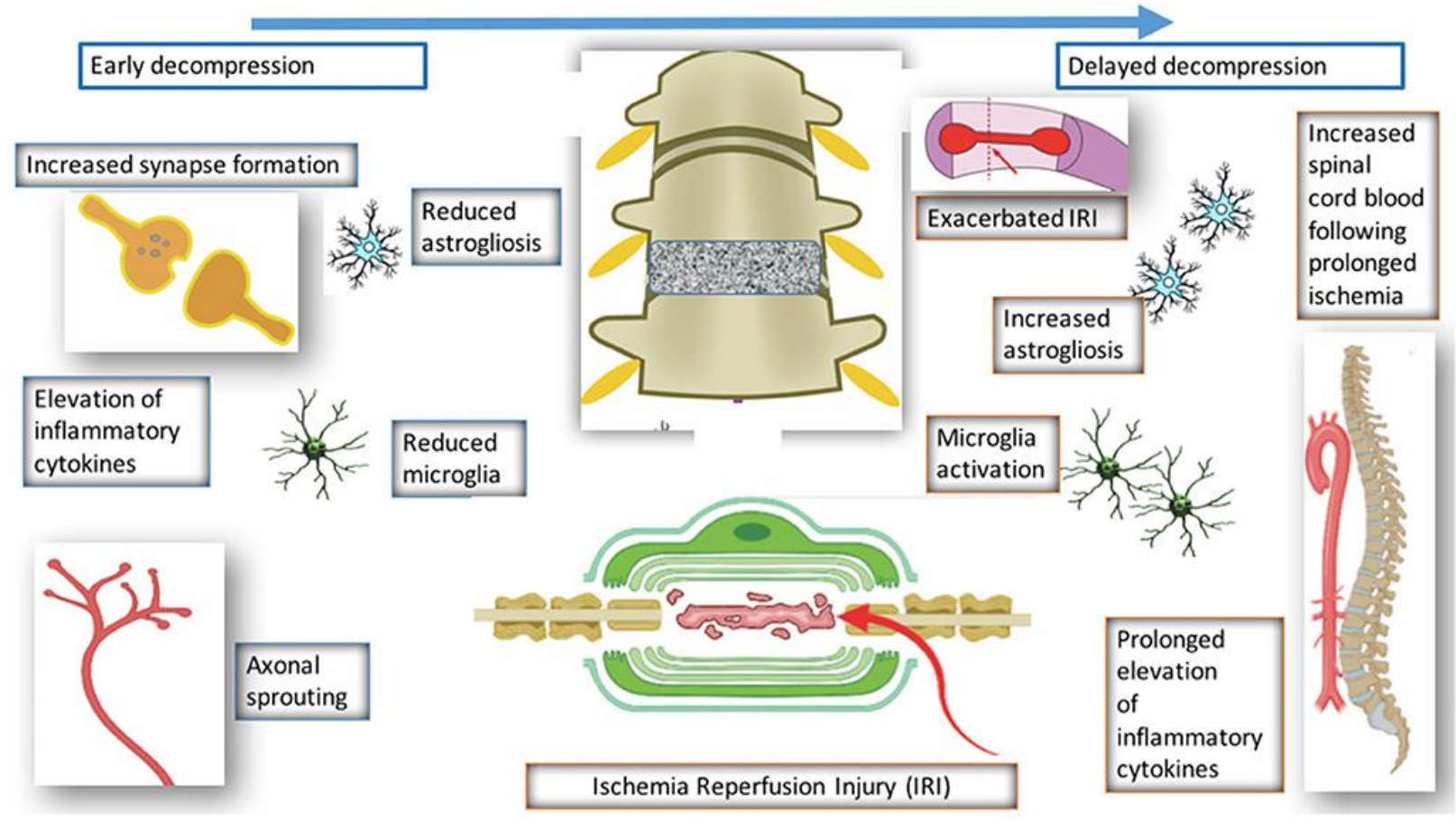

Figure 1: Pathological changes due to compression

\section{Clinical Features}

Symptoms can vary from mild to severe. You may have a flare up of symptoms if you over- use your neck, or if you sprain a neck muscle or ligament. Symptoms include:

Pain in the neck: This may spread to the base of the skull and shoulders. Movement of theneck may make the pain worse. The pain sometimes spreads down an arm to a hand or fingers. This is caused by irritation of a nerve which goes to the arm from the spinal cord in the neck. The pain tends to wax and wane with flare-ups from time to time. However, some people develop chronic (persistent) pain. 
Some neck stiffness: particularly after a night's rest.

Headaches from time to time: The headaches often start at the back of the head just the conditions which can produce a Lhermitte's (Lhermitte's phenomenon is named for French neurologist Jean Lhermitte) signs are:

1. Multiple sclerosis

2. Cervical spondylosis

3. Cervical disc herniation

4. Cervical spinal cord tumor

5. Chiari I malformation

6. Radiation myelopathy

7. Subacute combined degeneration (caused by vitamin B 12 deficiency)

\section{Investigation}

a) $x$-rays are useful for identifying such problems as:

- Narrowing of the intervertebral disc space

- Anterior osteophytes (i.e. bony spurs)

- Spondylosis (i.e. arthritis) of the facet joints

- Osteophytes from the uncovertebral joints (see figures below)

b) Computed Tomography: Computed tomography (CT) can highlight the bony changes associated with degenerative spondylosis (arthritis). Osteophytes can be observed and evaluated as well. However, CT does not provide for optimal evaluation of discs (although it may sometimes show disc herniations).

c) Magnetic Resonance Imaging: Magnetic resonance imaging (MRI) is a powerful tool in the assessment of patients with cervical spondylosis. Images from MRl's can help doctors to identify disc herniations, osteophytes and joint arthrosis. MRI is best suited for soft disc herniations, but often times more information is needed.

d) Myelogram/Ct: This is the "gold standard". It is often utilized in complex cases involving multilevel disease, or suboptimal MRI images.it is very useful in delineating bone spurs fromsafe disc herniations.

e) Discography: As in the lumbar and thoracic spine, cervical discography (see figure) remains controversial. Although the discogram may add to the children's knowledge, it should not be used by itself to predicate treatment.

\section{Management}

After conducting the necessary tests to identify the problem in the cervical spine, a treatment plan will then be developed. Various treatment options are available, and can be subdivided into two categories:

Non-operative treatment

$\square$ Operative treatment 


\section{Non-operative treatment}

It is believed in Allopathic system of medicine that non-operative treatment of cervical degenerative disease provides good to excellent results in over $75 \%$ of patients. A multidisciplinary approach includes:

- Immobilization can be achieved using a collar or braces; most beneficial during acute exacerbations of pain by reducing motion at the symptomatic levels.

- Physical therapy consists of traction, Diathermy and manipulation (chiropractic) can beuseful in decreasing muscle spasms that can contribute to symptoms; this is where heat, electrical stimulation, and exercise have their maximum benefit.

- Medications including painkillers, non-steroidal anti-inflammatory, and muscle laxants. In many cases, non operative treatment can provide good long-term results.

- Homoeopathic system of medicine provides a complete cure of this problem. The most common Homoeopathic remedies used in such cases are:

Exercise your neck and keep active: Aim to keep your neck moving as normally as possible. As far as possible, continue with normal activities.

Other advice:

A good posture may help. Brace your shoulders slightly backwards, and walk 'like a model'. Try not to stoop when you sit at a desk. Sit upright.

A firm supporting pillow seems to help some people when sleeping.

Physiotherapy. Therapies such as traction, heat, cold, manipulation, etc, may be tried when you have a flare-up of pain. However, the evidence that these help is not strong. What may be most helpful is the advice a physiotherapist can give on neck exercises to doat home.

\section{Homeopathic Approach for the Treatment of CervicalSpondylosis}

The treatment of CERVICAL SPONDYLOSIS by Homoeopathy has a very good scopeand favorable outcome. Unlike modern treatment which has many limitations to treat this conditions, we have a whole lot of medicines for its treatment.

Acute onset of cervical pain is mainly due to cervical spondylosis. As Master Hahnemann, hasstated in aphorism no 5 that 'exciting cause' is the most probable causative factor of acute diseases, so at first, we have to look for the exciting cause. The exciting cause of neck pain may be trauma, sprain, strain, exertion, exposure to cold etc. the above-mentioned causes may be solely responsible for neck pain if of sufficient intensity.

But in cases where there are underlying constitutional derangements any trivialexciting factor may cause same degree of neck pain.

Neck pain of chronic character are almost always due to fundamental cause which is generally due to a chronic Miasm; Psora, Syphilis and Sycosis. So, to investigate the fundamental cause the ascertainable physical constitution of the patient, his moral and intellectual character, his occupation, mode of living and habits, his social and domestic relations, his age, sexual function, etc are to be taken into consideration. Apart from the fundamental cause the maintaining cause should also be investigated. 
The basis of Homoeopathic prescription is individualization which again depends upon correct evaluation of symptoms. In the process of evaluation, the exciting, maintaining and fundamental cause of disease are of supreme importance and thereafter the rare, uncommon, peculiar and characteristic symptoms of the patient. In some cases, we get some uncommon symptoms of the disease and a few characteristic symptoms of the patients. The knowledge of pathology helps us to differentiate between common and uncommon symptoms of the disease and peculiar characteristic symptoms of the patient.

We can treat the patient in various ways. Homoeopathic prescription can be done on the following basis-

- According to totality of symptoms.

- According to constitution.

- According to etiology.

- According to pathological basis.

- According to tauopathy.

- According to Miasm.

- According to keynote symptoms.

Apart from these, sometimes in treatment of neck pain an intercurrent or blockremoval medicine may be needed to cure a chronic case. Palliative treatment can be given in some selected cases as in; cases with advanced and irreversible structural change, patient lacking in any vital organs, most urgent cases where danger to life and imminent death allows no time for the action of Homoeopathic medicine, as in accidents, spondylolisthesis. Finally, lam focusing my view on the treatment of neck pain. Different physicians have different angle of treatment. But in my opinion one should be open minded to treat any disorder.

\section{Homoeopathic Therapeutics}

Nevertheless, Homoeopathic prescription is based on individualization i.e. it cannot be prescribed successfully by some specific indication of some remedy although some of the indications of different drugs are given below. We must not follow solely the given indicationsbut we must prescribe based on totality of symptoms. There are countless drugs which cover low back pain. Most important drugs with indications are given below-

1. Agaricus muscaris: - sore aching pain in cervical regions, during exertion in the daytime, $<$ while eating, in cold weather, before thunderstorms. Single vertebra sensitive to touch.

2. Arnica Montana: - neck pain from over exertion and straining, every part is bruised, lame and sore. Trauma on neck. Everything on which he lies seems too hard, whole body oversensitive but patient says there is nothing the matter with him.

3. Bellis perennis: - it is a very good remedy for neck pain. Bad posture, sprain or strainmay be the cause. Railway spine, auto traumatism. Especially useful for gardeners. Great soreness, insomnia at 3 a.m.

4. Bryonia: - Painfull stiffness of neck worse by sudden change in weather, movement. Better by hard pressure, lying on painful side and cold things.

5. Calcarea carbonicum: - stiffness and rigidity at the nape of the neck in morning on turning head. Burning pain in the nape of the neck to occiput, lasting all day and ceasing only on going to sleep at night. Rheumatic pain in the upper cervical vertebrae with stiffness of neck. Easily overstrains himself from lifting, from which neck becomes stiff and rigid with headache. Pain between the shoulders and in the lower part of back, drawing pain between the shoulder blades. Dull shocks upwards in between shoulder blades, from posterior wall of chest. Cervical spondylitis. Most successful remedy in treating cervical 
spondylosis.

6. Calcarea flouricum: - neck pain from strains; pains < after rest, > from moving a little and from warmth (after failure of Rhustox). Features like Calcarea group and Fluoric acid.

7. Calcarea phoshorica: - useful in cervical spondylosis. Pain from draught of air. Soreness in cervical region as if broken. Violent pain in neck from slightest bodily effort. Spine weak disposed to curvatures, especially to the left. Unable to support head. Pain< autumn and cold weather, > spring. To unite fracture bone.

8. Cimicifuga racemosa:- rheumatic pain in the muscles of neck and back; feels stiff lame and contracted, spine sensitive, from using arms in sewing typewriting, piano playing. Muscular rheumatism. Crick in cervical region. The active principle Macrotin is used especially in neck pain.

9. Guiacum: - rheumatic stiffness of whole left side of the neck. Contraction of limbs, stiffness and immobility. Feeling that he must stretch. < Motion heat, cold wet weather.> External pressure. Aversion to milk; secretion are free and foul.

10. Hypericum: - after a fall, slightest motion of arms and neck, extorts cries, violent pains and inability to walk or stoop after a fall on coccyx. Spinal injury. Pain remains after spinal anaesthasia.

11. Gnaphalium:- chronic muscular rheumatism of back and neck.

12. Lachnantes:- rheumatism of neck. Stiffness of neck. Pain in nape, as if dislocated. Severe muscle spasm so much so that head is drawn to one side.

13. Lycopodium:- pains are tearing, often on right side, with or without swelling. In neck pain if Bryonia has not significantly relieved and the pain is worse from slightest motion. A constitutional remedy, if indicated acts infalliably.

14. Magnetis Poli Ambo: - painful stiffness in the cervical vertebrae in morning, during motion. Crackling in the cervical vertebrae in morning during motion.

15. Magnetis Polus Arcticus: -crackling or cracking in the cervical vertebrae especially in the atlas, during motion. Pain and bruised feeling in the middle of spine, when bending the spine backward. Gurgling and creeping sensation between the scapula.

16. Ustilago: - pain in the back of neck < by walking.

17. Medorrinum: -for the persons suffering from gout, rheumatism. Pains arthritic rheumatic, a sequel of suppressed gonorrhoea. Constiction seems to tighten whole body, sore all over bruised feeling. Pain in the back between the scapulae. Whole length of the spine sore to touch. Intense burning pain beginning in nape of neck and extending down spine, with a contractive stiffness, < by stretching

18. Oreodaphne:-cervico-occipital pain. Constant dull ache in the cervical and occipital region, extending to scapula down spine, into head. Constant desire to move the head which does not relieve.

19. Paris Quadrifollia: -sense of weight and weariness in nape of neck and across shoulders. Arms become stiff, fingers clenched. Fingers often feel numb. Usually on the left side.

20. Rhododendron: -joints swollen. Stiffness of the neck. Pain in shoulders, arms and wrist worse when at rest. Pain in the bones in spots reappear by change of weather.

21. Rhus Toxicodendron:-drawing pain in the fibrous tissues, joints and sheaths of nerves, right side more than the left; caused by exposure to wet damp weather, to rain.< Rest, first motion; >motion, continuous motion, warm application. Restlessness cannot remain in bed must change position often to obtain relief from pain. Desire for milk.

22. Ruta Graveolens:-lameness and sprains. Neck pain relieved by pressure and lying on back < moving. Injury to spinal processes (Periosteum).Bruised lame sensation all over, as after a fall or blow; worse in limbs and joints.

23. X Ray:- stiffness on left side of the neck, turning in bed. Stiffness on right side of neck, with intense pain at night; occurs in paroxysms during the day > somewhat by hot application. Sudden cricks attack first on the right side of the neck, then the other side $<$ on getting cold, turning the head nearly produces convulsions. Pains more severe behind the ears-the 
mastoid process.

24. Zincum Metalicum:-Nape of neck weary from writing or any exertion. Trearing in shoulder blades. Cannot bear back touched. Tension and stinging between shoulder blades.

\section{Conclusion}

Cervical spondylosis is very much growing disease in this modern era. There are lots of therapy \& medication used in conventional method, but it gives patient for relief of sometime. In Homoeopathy, there is lots of medicine for such kind of disease. We have lots of medicine to relief pain \& reduce its reoccurance. For such kind of disease, we must follow management, exercise, and diet along with medication which gives a very pleasant result.

\section{Bibliography}

[1] Davidson Stanley. Davidson's Principles and Practice of Medicine. 20th Edition. China: Churchill Livingstone Elsevier; 2006. p.1221-1222, 1241-1242.

[2] Das P.C. Textbook of Medicine: Nervous system. Kolkata: Current Books International; Jan 2000. p.478-479.

[3] Das Somen. The Spine and Pelvis. A Concise Textbook of Surgery. Fourth Edition: p.517518.

[4] The Associations of Surgeons of India. Orthopaedic Surgery-I Diseases of Bones and Joints. Textbook of Surgery: p.953-954.

[5] Hahnemann S. Organon of Medicine, $5^{\text {th }} \& 6^{\text {th }}$ ed. New Delhi: B. Jain publishers (P) Ltd.; 2016.

[6] Allen HC, Allen's Keynote Rearranged \& classified, $10^{\text {th }}$ ed. New Delhi: B. Jain publishers $(P)$ Ltd.; 2013.

[7] Boericke W. Boericke's New Manual of Homoeopathic Materia Medica \& Repertory, LPE ed. New Delhi: B. Jain Publishers (P) Ltd.; 2014. 